\title{
Management of Late Preterm and Term Neonates exposed to maternal Chorioamnionitis
}

\author{
Mitali Sahni ${ }^{1,4^{*}}$, María E. Franco-Fuenmayor ${ }^{2}$ and Karen Shattuck ${ }^{3}$
}

\begin{abstract}
Background: Chorioamnionitis is a significant risk factor for early-onset neonatal sepsis. However, empiric antibiotic treatment is unnecessary for most asymptomatic newborns exposed to maternal chorioamnionitis (MC). The purpose of this study is to report the outcomes of asymptomatic neonates $\geq 35$ weeks gestational age (GA) exposed to MC, who were managed without routine antibiotic administration and were clinically monitored while following complete blood cell counts (CBCs).

Methods: A retrospective chart review was performed on neonates with $G A \geq 35$ weeks with $M C$ during calendar year 2013. IT ratio (immature: total neutrophils) was considered suspicious if $\geq 0.3$. The data were analyzed using independent sample T-tests.

Results: Among the 275 neonates with MC, 36 received antibiotics for possible sepsis. Twenty-one were treated with antibiotics for $>48 \mathrm{~h}$ for clinical signs of infection; only one infant had a positive blood culture. All 21 became symptomatic prior to initiating antibiotics. Six showed worsening of IT ratio. Thus empiric antibiotic administration was safely avoided in $87 \%$ of neonates with MC. $81.5 \%$ of the neonates had follow-up appointments within a few days and at two weeks of age within the hospital system. There were no readmissions for suspected sepsis.
\end{abstract}

Conclusions: In our patient population, using CBC indices and clinical observation to predict sepsis in neonates with MC appears safe and avoids the unnecessary use of antibiotics.

Keywords: CBC, Newborn, Sepsis, Antibiotics

\section{Background}

Chorioamnionitis or intraamniotic infection is defined as acute inflammation of the membranes and chorion of the placenta, typically due to ascending polymicrobial bacterial infection. Most commonly, chorioamnionitis is associated with ruptured membranes, but organisms including Group B streptococcus, Ureaplasma species, Mycoplasma hominis, and Listeria monocytogenes infect intact membranes as well. MC is a significant risk factor for early-onset sepsis (EOS) due to Group B streptococcus (GBS) and can reflect an intrauterine onset of infection in the neonate $[1,2]$. The traditional diagnostic criteria for clinical chorioamnionitis

\footnotetext{
*Correspondence: mitali12@gmail.com

${ }^{1}$ Division of Neonatology, Drexel University College of Medicine, Philadelphia, PA, USA

${ }^{4}$ St. Christopher's Hospital for Children, 160 East Erie Avenue, Philadelphia, PA 19134, USA

Full list of author information is available at the end of the article
}

include maternal fever $\left(>100.4^{\circ} \mathrm{F}\right.$ persisting more than $1 \mathrm{~h}$ or any fever more than $101^{\circ} \mathrm{F}$ ), and two or more of the following: fetal tachycardia ( $>160 / \mathrm{min})$, maternal tachycardia ( $>100 / \mathrm{min}$ ), uterine tenderness, and foul-smelling or purulent amniotic fluid.

To address possible EOS in asymptomatic newborns with MC, the 2010 American Academy of Pediatrics (AAP) and Center for Disease Control and Prevention (CDC) recommend a limited evaluation including blood culture (at birth) and CBC with differential (at birth and/or at $6-12 \mathrm{~h}$ of life (HOL) along with initiation of empiric antibiotics. However, a recent epidemiological study found that among term infants exposed to MC, only $13.5 \%$ received antibiotics after birth [3]. This deviation from guidelines, as seen in practice, has been attributed to improved obstetrical interventions, which significantly decreased the risk of sepsis in late preterm and term neonates in this

(C) The Author(s). 2019 Open Access This article is distributed under the terms of the Creative Commons Attribution 4.0 International License (http://creativecommons.org/licenses/by/4.0/), which permits unrestricted use, distribution, and 
setting [4]. A multispecialty workshop sponsored by the Eunice Kennedy Shriver National Institute of Child Health and Human Development, recommended treating late preterm and term neonates only if the diagnosis of chorioamnionitis is confirmed with pathology or microbiology [5]. The American College of Obstetricians and Gynecologists (ACOG) supported this and introduced the term intraamniotic infection for chorioamnionitis. They further defined suspected intraamniotic infection as maternal intrapartum fever (single maternal temperature $\geq 39.0^{\circ} \mathrm{C}$ or a temperature of $38.0^{\circ} \mathrm{C}-38.9^{\circ} \mathrm{C}$ that persists for $>30 \mathrm{~min}$ during labor) presenting with 1 or more of the following: purulent cervical drainage, maternal leukocytosis, and fetal tachycardia [6]. The most recent AAP statement addressing management of neonates born at $\geq 35$ weeks GA at risk for EOS suggests utilizing a locally tailored guideline by birth centers, for EOS risk assessment and clinical management [7]. They also suggested utilizing multivariate risk assessment tools like the Neonatal Early Onset Sepsis Risk Calculator to assess the risk of EOS [7-9]. In our hospital, the practice is to perform CBCs at 6 and $24 \mathrm{~h}$ after birth, and initiate the sepsis work-up and antibiotics (Ampicillin and Gentamicin) only if the baby develops signs/symptoms of sepsis or the CBCs suggest infection. Since our practice was initiated, others have looked at the management of neonates exposed to $M C$ without antibiotics $[10,11]$. The purpose of this study is to report our observational practice of managing asymptomatic neonates $\geq 35$ weeks GA exposed to $M C$ without empiric antibiotic administration. In addition, we include follow-up of these neonates after hospital discharge to evaluate the safety of our practice.

\section{Methods}

A retrospective chart review was done on all neonates with GA $\geq 35$ weeks, who were exposed to MC. The study included 275 neonates over 12 months (January to December 2013). The institutional review board at the University of Texas Medical Branch (UTMB) at Galveston approved this study. In November 2012, we instituted a practice guideline for management of asymptomatic neonates exposed to maternal chorioamnionitis, using serial CBCs and close observation. For the study, MC was defined as maternal treatment with antibiotics for chorioamnionitis. Data collected included the following:

- Neonatal demographic data included birth weight, gender, and gestational age.

- Maternal data including maternal age, GBS status and antibiotic treatment before delivery.

- Results of CBC with differential at 6 and 24 HOL, including the ratio of immature to mature neutrophils (I/T ratio), absolute neutrophil count (ANC), and total leukocyte count (TLC). The differential count is obtained manually by trained technicians and confirmed by hematologists. I/T ratio was considered suspicious if $\geq 0.3$.

- Presence of signs and symptoms of sepsis including respiratory distress (tachypnea: respiratory rate $>60 /$ min, increased work of breathing or need for oxygen), temperature instability (Temperature $<36.5$ $C$ that could not be attributed to environmental factors), hypoglycemia and feeding difficulty (poor feeding with low urine output).

- The incidence of sepsis work-up and transfer to neonatal intensive care unit (NICU).

- Results of blood culture and time interval for it, organisms isolated, length of antibiotic use and hospital stay.

- Chart review for follow up visit 2-3 days after discharge and at two weeks of age.

- Chart review for readmission after discharge to rule out sepsis.

The data collected were analyzed using descriptive statistics and independent sample T-tests. SPSS software platform was utilized to do the statistical analysis. $P$ value $<0.05$ was considered significant.

\section{Results}

In 2013, 5006 babies $\geq 35$ weeks gestation were born at UTMB. Two hundred seventy-five were born to mothers with a diagnosis of chorioamnionitis, for an incidence of $5.49 \%$. At least two CBCs were obtained on all neonates with MC. The baseline characteristics of the study population are presented in Table 1. One hundred sixty (58.2\%) of the infants were born via vaginal delivery. Of the 275 mothers with chorioamnionitis, the majority were not colonized with GBS. Of those who were positive for GBS, 90\% were treated adequately. Table 2 illustrates the GBS status of the mothers and the information about their treatment. $68.4 \%$ of the mothers with the diagnosis of $\mathrm{MC}$ were administered antibiotics at least one hour before delivery.

The first $\mathrm{CBC}$ was obtained at a mean of $5.85 \mathrm{HOL}$ (range 0-12, mode 6). The mean ANC was 14.9 \pm 5.5 , and the mean $\mathrm{I} / \mathrm{T}$ ratio was $0.29 \pm 0.15$ at this time. One hundred sixty-three infants (40.7\%) had a suspicious I/T ratio (>0.3) on the first CBC. Only 1 out of the 163 infants with

Table 1 Baseline characteristics of the study population

\begin{tabular}{ll}
\hline Baseline characteristics of the study population \\
\hline Mean gestational age (weeks) & 38.8 wks (range 35-41wks) \\
Birth weight (grams) & $3368 \mathrm{~g}$ (range 2070-5029 g) \\
Male (\%) & 51.3 \\
Infants born at $\geq 35$ weeks & 5006 \\
gestation age (2013) & \\
Incidence of MC in infants & 5.49 \\
$\geq 35$ weeks gestation (\%) & \\
\hline
\end{tabular}


Table 2 GBS status of mothers with chorioamnionitis

\begin{tabular}{ll}
\hline GBS status & Percentage of mothers \\
\hline Negative & $84.4 \%$ \\
Positive- Adequately treated & $10.2 \%$ \\
Positive- Not treated & $1.1 \%$ \\
Unknown- Adequately treated & $1.5 \%$ \\
Unknown- Not treated & $2.9 \%$ \\
\hline
\end{tabular}

increased I/T ratio at the time of first $\mathrm{CBC}$ was diagnosed with EOS with a positive blood culture. The positive predictive value (PPV) for early $\mathrm{I} / \mathrm{T}$ ratio $(>0.3)$ to diagnose EOS was $0.61 \%(95 \%$ CI $0.56-0.68 \%)$. The second CBC was obtained at a mean of $22 \mathrm{HOL}$ (range 7-32, mode 24). Mean ANC was $12.16 \pm 4.8$ and mean I/T ratio $0.18 \pm 0.15$. Fewer infants $(18.54 \%)$ had a suspicious $\mathrm{I} / \mathrm{T}$ ratio on the second $\mathrm{CBC}$. The PPV of $\mathrm{I} / \mathrm{T}$ ratio (>0.3) to diagnose EOS at the time of the second CBC was 1.96\% (95\% CI 1.532.51\%). In 48 infants the $\mathrm{I} / \mathrm{T}$ ratio increased at the time of second $\mathrm{CBC}$. This increase in IT ratio was not predictive of change in clinical presentation as only 6 out of these 48 infants had clinical signs of sepsis. Twenty-two of these infants had an initial $\mathrm{CBC}$ with $\mathrm{I} / \mathrm{T} \geq 0.3$.

Not all neonates with an increase in $\mathrm{I} / \mathrm{T}$ ratio were transferred to the NICU for further workup. The decision to transfer was made based on the overall clinical presentation of the infant and attending's judgment. Thirty-six infants (13.1\%) were transferred to the NICU for further evaluation including blood culture and antibiotics. Although only one infant had a positive blood culture, 21 infants $(7.6 \%)$ were treated with antibiotics for more than $48 \mathrm{~h}$ due to concern for clinical sepsis (Fig. 1). The duration of antibiotics in these infants ranged from 3 to 7 days and was dependent on clinical judgment of the attending. The infant with a positive blood culture was born via vaginal delivery at 35 weeks GA and developed respiratory distress within the first $6 \mathrm{~h}$ after birth. She was transferred to the NICU due to clinical suspicion for EOS and was found to have a high I/
$\mathrm{T}$ ratio of 0.72 and 0.48 at the time of the first and second $\mathrm{CBC}$, respectively. She was treated with antibiotics for 10 days for culture positive sepsis. Infant's mother was diagnosed with $\mathrm{MC}$ based on high maternal temperature $\left(101^{\circ} \mathrm{F}\right)$ along with fetal and maternal tachycardia. All neonates treated with $>48 \mathrm{~h}$ of antibiotics developed clinical signs of sepsis (respiratory distress, poor feeding or hypothermia) and $28 \%$ of these neonates had an increase in the I/T ratio. Figure 1 shows the percentage of infants with $\mathrm{MC}$ who received further management.

An independent-sample t-test was conducted to compare the $\mathrm{CBC}$ indices in the neonates who were treated with antibiotics for $>48 \mathrm{~h}$ with neonates who were either not treated or treated for $\leq 48 \mathrm{~h}$ (Table 3). Both IT ratios differed between the group treated with antibiotics $>48 \mathrm{~h}$ vs. those treated for $\leq 48 \mathrm{~h}$ and untreated group $(p=0.003$ for the first CBC, $p=0.002$ for the second $\mathrm{CBC}$ ). At the time of first $\mathrm{CBC}$, rupture of membrane $(\mathrm{ROM})>18 \mathrm{~h}$ was associated with a higher likelihood of a suspicious IT ratio $(p=0.023)$. There were no significant differences with regard to gender, birth weight, mode of delivery or presence of respiratory distress.

All newborns are scheduled for follow-up appointments at 2-3 days and 2-weeks after discharge. Two hundred twenty-four neonates (81.5\%) attended the 2-3 days appointment and $226(82.2 \%)$ were seen at two weeks of age at a clinic within our hospital system where a full history and physical exam was obtained. At the time of follow up visits, none of these infants exposed to maternal chorioamnionitis demonstrated any concern for sepsis. Furthermore, none of the infants in our study were readmitted to our hospital for evaluation of sepsis.

\section{Discussion}

Maternal chorioamnionitis is a well-known risk factor for EOS; however, there is considerable variation in the accepted definition and diagnosis of MC. Frequently it is used to describe a heterogeneous group of conditions

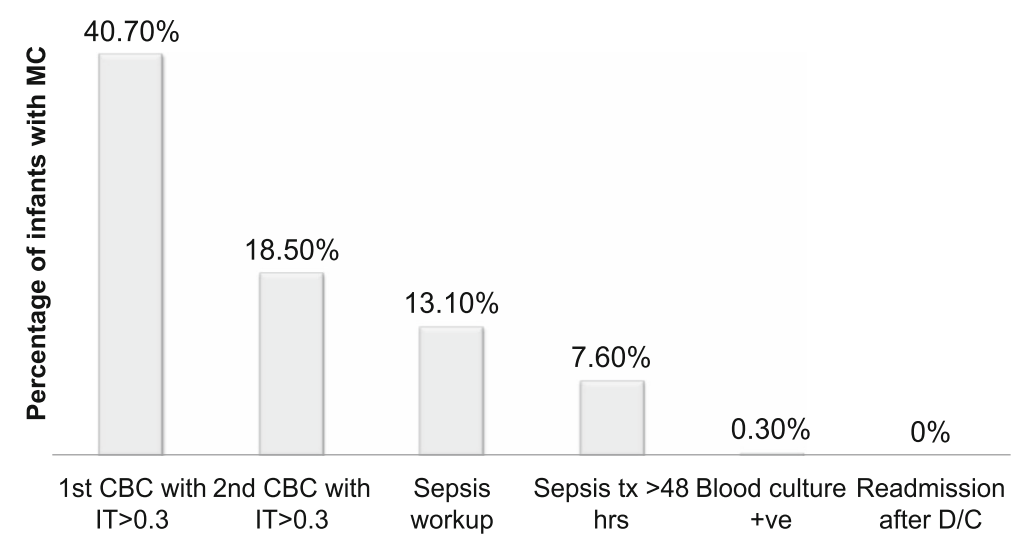

Fig. 1 Progression of management of infants exposed to maternal chorioamnionitis 
Table 3 ANC and IT ratio significance (*Mean \pm S.D.)

\begin{tabular}{|c|c|c|c|}
\hline Variables & $A b x>48 h$ & No $\mathrm{Abx} / \mathrm{Abx} \leq 48 \mathrm{~h}$ & $P$ value $[95 \% \mathrm{Cl}$ for difference between means] \\
\hline IT ratio* (1st CBC) & $0.45 \pm 0.24$ & $0.27 \pm 0.14$ & $0.003[0.07$ to 0.29$]$ \\
\hline IT ratio* (2nd CBC) & $0.38 \pm 0.25$ & $0.16 \pm 0.13$ & $\mathbf{0 . 0 0 2}[0.08$ to 0.32$]$ \\
\hline $\mathrm{ANC}^{*}(1 \mathrm{st} \mathrm{CBC})$ & $10.55 \pm 4.9$ & $15.32 \pm 5.4$ & $<0.001[-7.1$ to -2.4$]$ \\
\hline $\mathrm{ANC}^{*}(2 \mathrm{nd}$ CBC) & $14.05 \pm 6.7$ & $12.01 \pm 4.6$ & $0.197[-1.1$ to 5.2$]$ \\
\hline
\end{tabular}

Difference in CBC indices between the groups treated with prolonged antibiotics for a diagnosis of clinical sepsis vs. the group not treated with antibiotics and treated for $48 \mathrm{~h}$ or less. ANC: absolute neutrophil count; Abx: antibiotics; Significant $p$ values are presented in bold

ranging from sterile inflammation to infection of varying degree of severity and duration. Some centers diagnose $\mathrm{MC}$ by the presence of intrapartum fever alone (unexplained by other infection), while others favor a diagnosis described by Gibbs et al. [12] that requires presence of intrapartum fever of at least $100{ }^{\circ} \mathrm{F}\left(37.8^{\circ} \mathrm{C}\right)$ as well as at least two additional clinical signs (maternal tachycardia $>100$ beats per minute, fetal tachycardia $\geq 160$ beats per minute), uterine tenderness, foul-smelling amniotic fluid, and leukocytosis $\geq 15,000$ cells $/ \mathrm{mm}^{3}$ ) [13]. There are other variations of this definition also in use, and hence the term chorioamnionitis does not consistently reflect the severity of maternal and fetal illness [14]. In our setting, the obstetricians assess the risk for chorioamnionitis using the clinical criteria proposed by Gibbs et al. described above. Antibiotics are begun only if they make the clinical diagnosis. Our setting is unusual in that the obstetricians (all in the academic maternal-fetal medicine group) have a unified approach to the diagnosis and treatment. The babies born to those mothers are the subject of this report. We do not have a histological diagnosis for these cases.

The 2010 guidelines by the CDC [15] and AAP [16] recommend treatment of all neonates exposed to $\mathrm{MC}$ irrespective of gestational age and presence of symptoms. As a consequence of these guidelines, many term and late preterm neonates are unnecessarily exposed to antimicrobial agents in order to prevent rare cases of EOS. Surveillance studies estimate the incidence of EOS to be 0.77 cases/ 1000 live births. This can be further broken down to $\sim 0.5$ cases/1000 among those born at $\geq 37$ weeks, compared to $\sim 3.0$ cases/1000 live births occurring at $<37$ weeks gestation [17]. However, the presence of chorioamnionitis increases this risk by 2-3 fold [18]. Reported rates of confirmed EOS in infants born at $\geq 35$ weeks' gestation to mothers with clinical chorioamnionitis range from 0.47 to $1.24 \%[4,14]$. This risk of EOS is reduced if symptomatic newborns are excluded from the analysis $[4,19]$. In a multicenter, prospective surveillance study EOS was diagnosed in 389 of the 396,586 infants reviewed; $60 \%$ of these infants were exposed to $\mathrm{MC}$. The researchers estimated that almost 450 term infants exposed to MC would have to be treated for every case of confirmed EOS [20]. Despite being a relatively small sample size, the data from our study is consistent with the observation that the risk of EOS is low in infant exposed to MC (1 case of EOS in 275 infants, $0.36 \%)$. There were no cases of EOS in infants that were exposed to $\mathrm{MC}$ who remained asymptomatic within the first $24 \mathrm{~h}$. Babies who were $<35$ weeks or were clinically ill at birth were admitted directly to the NICU and are not addressed in this report.

A multivariate risk assessment model to predict the risk of EOS, was developed using objective data collected at the time of birth [21] and the evolving newborn condition during the first 6 to $12 \mathrm{~h}$ after birth [22]. The objective data included the highest maternal intrapartum temperature, the maternal GBS colonization status, the type and duration of intrapartum antibiotic therapies, the duration of ROM and gestational age at the time of delivery. One web-based Neonatal Early-Onset Sepsis Risk Calculator (https://neonatalsepsiscalculator.kaiserpermanente.org) uses a predictive model developed from a large population to calculate the risk estimate for EOS and recommend management [23]. The recent AAP statement regarding management of suspected/proven EOS in neonates $\geq 35$ weeks' gestation suggests utilizing the sepsis calculator in conjunction with serial physical exams every $4-6 \mathrm{~h}$ for the first $48 \mathrm{~h}$ [7]. At our center, we continue to use serial physical exams and clinical assessment to evaluate for risk of EOS in asymptomatic term infants. Use of multivariate risk assessment tool has not been implemented in practice.

In 2016, an Expert Panel Workshop summary by the ACOG questioned the current method of diagnosing chorioamnionitis and proposed the use of an alternate diagnosis of Triple I for pathological diagnosis of chorioamnionitis. They further recommended treating late preterm and term neonates only if the diagnosis of chorioamnionitis is confirmed with pathology or microbiology. This would involve the presence of clinical symptoms and any one of the following:

1. Positive gram stain on amniocentesis;

2. Low glucose or positive amniotic fluid culture; or

3. Placental pathology revealing infection [5].

However, this information is rarely available to use at the time of birth when the decision to do a sepsis workup is being made. In August 2017, the ACOG Committee on 
Obstetric Practice released a committee opinion statement about evaluation and management of intrauterine infection [6], which clarified their earlier recommendations and suggested the use of multivariate risk assessment and increased reliance on clinical observation to safely decrease the number of well-appearing term newborns being treated empirically with antibiotics. However, they did not include Triple I as a new diagnostic category.

Antibiotic therapy in late preterm and term neonates born to mothers with MC ideally should be restricted to those who are at increased risk of developing EOS. Unfortunately, no diagnostic test effectively predicts the infants at risk. Hence there are different approaches used by different centers to limit the unnecessary use of antibiotics in this setting. Some centers use C-reactive protein in addition to $\mathrm{CBC}$ indices to predict the risk of EOS [10, 19, 24] and some use the neonatal EOS calculator to help with management of these neonates $[9,11]$. Most neonates at UTMB are born to mothers who receive prenatal care in a system of Regional Maternal and Children's clinics, which is staffed by members of the $\mathrm{OB}$ faculty. Thus, we have the advantage of large delivery service with a consistent practice of screening for GBS and a standard definition of MC. At our center, we perform a sepsis evaluation on the basis of $\mathrm{CBC}$ indices and presence of other risk factors including duration of rupture of membranes, the severity of maternal illness and maternal GBS colonization.

Historically, CBC indices such as WBC count, ANC, IT ratio and platelet counts have been used to predict the risk of EOS. Hornik et al. studied 166,092 infants and found that low WBC count, low ANC, and high IT ratios were associated with increased odds of infection (highest odds ratios: 5.38, 6.84, and 7.97, respectively). They also found that the specificity and negative predictive values of using CBC indices were high (73.7-99.9 and $>99.8 \%$, respectively). However, sensitivities were low $(0.3-54.5 \%)$ for all complete blood cell count indices analyzed [25]. Based on these results they recommend against the sole use of $\mathrm{CBC}$ indices to rule out infection, but to continue monitoring for clinical signs of sepsis and maintaining a high index of suspicion for those infants at risk remains necessary. Another large retrospective cross-sectional study evaluated $\mathrm{CBC}$ indices for predicting the risk of sepsis and found that WBC count, ANC and IT ratio were better at discriminating the risk of infection after $4 \mathrm{~h}$ of birth as these indices can be affected by many other factors in the first few hours of birth [26]. Hence, as part of our clinical protocol, we obtain the first $\mathrm{CBC}$ at about $6 \mathrm{~h}$ of life in asymptomatic neonates. In the study by Hornik et al., elevated $\mathrm{I} / \mathrm{T}$ ratios $(>0.2,>0.25,>0.5)$ were associated with relatively high specificities $(73.7,81.7,95.7 \%$, respectively) and negative predictive values $(99.2,99.2,99.0 \%$, respectively) [25] to predict the risk of EOS. In our study, at the time of first $\mathrm{CBC}$, the lowest value of IT ratio was 0.19.
The higher IT ratio seen in our population may be attributed to inflammatory response associated with chorioamnionitis and frequently associated risk of prolonged ROM. Our analysis showed that I/T ratio $>0.3$ had a poor positive predictive value for diagnosis of EOS (0.61 and 1.96\% at the time of the first and second $\mathrm{CBC}$ respectively). $\mathrm{I} / \mathrm{T}$ ratios were not reliably predictive of the perceived need for antibiotic treatment, with a sensitivity of only $28 \%$ for infants with that outcome. Therefore our data supports the recommendation to not use $\mathrm{CBC}$ indices alone, to predict the risk of EOS.

Our study has some limitations. First, our sample size was relatively small. Since the incidence of EOS is relatively low, our study may not be powered to estimate the risk of not treating the neonates with MC. Second, clinical sepsis was treated for more than $48 \mathrm{~h}$ based on clinical presentation in most cases. Third, our follow up data was limited to $81.6 \%$ of neonates in the study, and cannot predict if any of those with lack of follow up within our system were admitted elsewhere for EOS. However, our center is the most common pediatric provider for babies born at UTMB, due to the characteristics of the population we serve.

\section{Conclusions}

In our population, we chose to monitor clinical status and $\mathrm{CBC}$ indices in neonates exposed to $\mathrm{MC}$ to avoid NICU admission, prolonged length of stay, separation from mothers, and antibiotics in a large group of neonates who would prove to be healthy. $\mathrm{CBC}$ indices should not be used alone to predict the risk of EOS. This study adds evidence to support the use of observation-based approaches for managing newborns exposed to maternal chorioamnionitis. Using clinical observation and laboratory evaluation, we were able to avoid these adverse events in $87 \%$ (239/275) of neonates exposed to MC. Our follow up data from neonates after discharge attests to the safety of observation-based approach for the management of maternal chorioamnionitis. Although this study is insufficiently powered to assure complete elimination of EOS, the current recommendations for close follow-up after discharge is a useful safety net.

\section{Abbreviations \\ AAP: American Academy of Pediatrics; ACOG: American College of Obstetricians and Gynecologists; ANC: absolute neutrophil count: CBC: complete blood cell count; CDC: Center for Disease Control and Prevention; EOS: early-onset sepsis; GA: gestational age; GBS: Group B Streptococcus; HOL: hours of life; MC: maternal chorioamnionitis; NICU: neonatal intensive care unit; ROM: rupture of membrane; TLC: total leukocyte count; UTMB: University of Texas Medical Branch}

\section{Acknowledgements}

Not applicable.

Authors' contributions

MS conceptualized and designed the study, collected the data, carried out the statistical analysis and drafted the initial manuscript. MFF assisted with 
data collection, statistical analysis and revised the manuscript. KS conceptualized and designed the study, coordinated and supervised data collection, reviewed and revised the manuscript, and approved the final manuscript as submitted. All authors read and approved the final manuscript.

\section{Funding}

No external funding.

\section{Availability of data and materials}

The datasets used and/or analyzed during the current study are available from the corresponding author upon reasonable request.

\section{Ethics approval and consent to participate}

The Institutional Review Board (IRB) at the University of Texas Medical Branch, Galveston, TX, approved the study. The need for written consent was waived by the IRB.

\section{Consent for publication}

Not applicable.

\section{Competing interests}

The authors declare that they have no competing interests.

\section{Author details}

'Division of Neonatology, Drexel University College of Medicine, Philadelphia, PA, USA. ${ }^{2}$ Department of Pediatrics, University of Texas Medical Branch, Galveston, TX, USA. ${ }^{3}$ Division of Neonatology, Department of Pediatrics, University of Texas Medical Branch, Galveston, TX, USA. ${ }^{4}$ St. Christopher's Hospital for Children, 160 East Erie Avenue, Philadelphia, PA 19134, USA.

Received: 3 January 2019 Accepted: 30 July 2019

Published online: 13 August 2019

\section{References}

1. Yancey MK, Duff $P$, Kubilis $P$, Clark $P$, Frentzen BH. Risk factors for neonatal sepsis. Obstet Gynecol. 1996:87(2):188-94.

2. Adams WG, Kinney JS, Schuchat A, Collier CL, Papasian CJ, Kilbride HW, Riedo FX, Broome CV. Outbreak of early onset group B streptococcal sepsis. Pediatr Infect Dis J. 1993;12(7):565-70.

3. Malloy MH. Chorioamnionitis: epidemiology of newborn management and outcome United States 2008. J Perinatol. 2014:34(8):611-5.

4. Benitz WE, Wynn JL, Polin RA. Reappraisal of guidelines for management of neonates with suspected early-onset sepsis. J Pediatr. 2015;166(4):1070-4.

5. Higgins RD, Saade G, Polin RA, Grobman WA, Buhimschi IA, Watterberg K, Silver RM, Raju TN. Evaluation and Management of Women and Newborns with a maternal diagnosis of Chorioamnionitis: summary of a workshop. Obstet Gynecol. 2016:127(3):426-36.

6. Committee Opinion No. 712: Intrapartum Management of Intraamniotic Infection. Obstet Gynecol 2017, 130(2):e95-e101.

7. Puopolo KM, Benitz WE, Zaoutis TE. Management of Neonates Born at $>/=$ 35 0/7 Weeks' gestation with suspected or proven early-onset bacterial Sepsis. Pediatrics. 2018

8. Carola D, Vasconcellos M, Sloane A, McElwee D, Edwards C, Greenspan J, Aghai ZH. Utility of early-onset Sepsis risk calculator for neonates born to mothers with Chorioamnionitis. J Pediatr. 2018;195:48-52 e41.

9. Ayrapetyan M, Carola D, Lakshminrusimha S, Bhandari V, Aghai ZH. Infants born to mothers with clinical Chorioamnionitis: a cross-sectional survey on the use of early-onset Sepsis risk calculator and prolonged use of antibiotics. Am J Perinatol. 2018.

10. Jan Al, Ramanathan R, Cayabyab RG. Chorioamnionitis and Management of Asymptomatic Infants $>$ /=35 weeks without empiric antibiotics. Pediatrics. 2017;140(1).

11. Money N, Newman J, Demissie S, Roth P, Blau J. Anti-microbial stewardship: antibiotic use in well-appearing term neonates born to mothers with chorioamnionitis. J Perinatol. 2017:37(12):1304-9.

12. Gibbs RS, Blanco JD, St Clair PJ, Castaneda YS. Quantitative bacteriology of amniotic fluid from women with clinical intraamniotic infection at term. J Infect Dis. 1982;145(1):1-8.

13. Avila C, Willins JL, Jackson M, Mathai J, Jabsky M, Kong A, Callaghan F, Ishkin S, Shroyer AL. Usefulness of two clinical chorioamnionitis definitions in predicting neonatal infectious outcomes: a systematic review. Am J Perinatol. 2015:32(11):1001-9.

14. Hooven TA, Randis TM, Polin RA. What's the harm? Risks and benefits of evolving rule-out sepsis practices. J Perinatol. 2018:38(6):614-22.

15. Verani JR, McGee L, Schrag SJ. Prevention of perinatal group B streptococcal disease--revised guidelines from CDC, 2010. MMWR Recommendations and reports: Morbidity and mortality weekly report Recommendations and reports / Centers for Disease Control. 2010, 59:Rr-10):1-36.

16. Polin RA. Management of neonates with suspected or proven early-onset bacterial sepsis. Pediatrics. 2012;129(5):1006-15.

17. Weston EJ, Pondo T, Lewis MM, Martell-Cleary P, Morin C, Jewell B, Daily P, Apostol M, Petit S, Farley M, et al. The burden of invasive early-onset neonatal sepsis in the United States, 2005-2008. Pediatr Infect Dis J. 2011; 30(11):937-41.

18. Alexander JM, McIntire DM, Leveno KJ. Chorioamnionitis and the prognosis for term infants. Obstet Gynecol. 1999;94(2):274-8.

19. Kiser C, Nawab U, McKenna K, Aghai ZH. Role of guidelines on length of therapy in chorioamnionitis and neonatal sepsis. Pediatrics. 2014;133(6):992-8.

20. Wortham JM, Hansen NI, Schrag SJ, Hale E, Van Meurs K, Sanchez PJ, Cantey JB, Faix R, Poindexter B, Goldberg R, et al. Chorioamnionitis and cultureconfirmed, early-onset neonatal infections. Pediatrics. 2016;137(1).

21. Puopolo KM, Draper D, Wi S, Newman TB, Zupancic J, Lieberman E, Smith $M$, Escobar GJ. Estimating the probability of neonatal early-onset infection on the basis of maternal risk factors. Pediatrics. 2011;128(5):e1155-63.

22. Escobar GJ, Puopolo KM, Wi S, Turk BJ, Kuzniewicz MW, Walsh EM, Newman TB, Zupancic J, Lieberman E, Draper D. Stratification of risk of early-onset Sepsis in newborns $\geq 34$ weeks' gestation. Pediatrics. 2014;133(1):30-6.

23. Kuzniewicz MW, Puopolo KM, Fischer A, Walsh EM, Li S, Newman TB, Kipnis P, Escobar GJ. A quantitative, risk-based approach to the management of neonatal early-onset Sepsis. JAMA Pediatr. 2017;171(4):365-71.

24. Smulian JC, Bhandari V, Vintzileos AM, Shen-Schwarz S, Quashie C, Lai-Lin $\mathrm{YL}$, Ananth CV. Intrapartum fever at term: serum and histologic markers of inflammation. Am J Obstet Gynecol. 2003;188(1):269-74.

25. Hornik CP, Benjamin DK, Becker KC, Benjamin DK, Li J, Clark RH, CohenWolkowiez M, Smith PB. Use of the complete blood cell count in earlyonset neonatal Sepsis. Pediatr Infect Dis J. 2012;31(8):799-802.

26. Newman TB, Puopolo KM, Wi S, Draper D, Escobar GJ. Interpreting complete blood counts soon after birth in newborns at risk for sepsis. Pediatrics. 2010; 126(5):903-9.

\section{Publisher's Note}

Springer Nature remains neutral with regard to jurisdictional claims in published maps and institutional affiliations.

Ready to submit your research? Choose BMC and benefit from:

- fast, convenient online submission

- thorough peer review by experienced researchers in your field

- rapid publication on acceptance

- support for research data, including large and complex data types

- gold Open Access which fosters wider collaboration and increased citations

- maximum visibility for your research: over $100 \mathrm{M}$ website views per year

At BMC, research is always in progress.

Learn more biomedcentral.com/submissions 